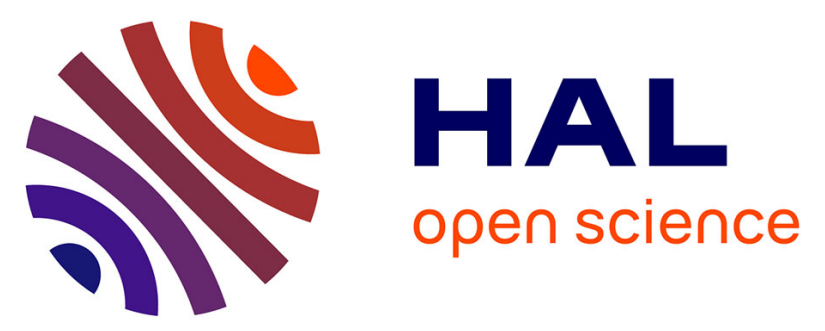

\title{
Three-dimensional magnetic structures generated by the development of the filamentation (Weibel) instability in the relativistic regime
}

Francesco Califano, Daniele del Sarto, Francesco Pegoraro

\section{- To cite this version:}

Francesco Califano, Daniele del Sarto, Francesco Pegoraro. Three-dimensional magnetic structures generated by the development of the filamentation (Weibel) instability in the relativistic regime. Physical Review Letters, 2006, 96 (10), pp.105008. 10.1103/PhysRevLett.96.105008 . hal-00524423

HAL Id: hal-00524423

https://hal.science/hal-00524423

Submitted on 30 May 2018

HAL is a multi-disciplinary open access archive for the deposit and dissemination of scientific research documents, whether they are published or not. The documents may come from teaching and research institutions in France or abroad, or from public or private research centers.
L'archive ouverte pluridisciplinaire HAL, est destinée au dépôt et à la diffusion de documents scientifiques de niveau recherche, publiés ou non, émanant des établissements d'enseignement et de recherche français ou étrangers, des laboratoires publics ou privés. 


\title{
Three-Dimensional Magnetic Structures Generated by the Development of the Filamentation (Weibel) Instability in the Relativistic Regime
}

\author{
F. Califano, D. Del Sarto, and F. Pegoraro \\ Physics Department and CNISM, University of Pisa, Pisa, Italy \\ (Received 25 November 2005; published 16 March 2006)
}

\begin{abstract}
We present three-dimensional, fully relativistic, fluid simulations of the dynamics of inhomogeneous counter streaming beams with the aim of understanding the magnetic structures that can be expected to form as a consequence of the development of the so-called Weibel instability. Ringlike structures in the transverse direction are generated as a consequence of the development of a spatially resonant mode. We describe the structures generated by beams of equal initial density and velocity and by a fast, less dense beam compensated by a slower, denser beam. We consider these two cases as schematic models of a laser produced beam propagating in a plasma with nearly equal density and in a plasma much denser than the injected beam.
\end{abstract}

DOI: 10.1103/PhysRevLett.96.105008

Fast, relativistic collimated electrons accelerated by the interaction of ultra strong laser pulses with an overdense plasma play a key role in the Fast Ignition approach to Inertial Confinement Fusion, where a petawatt-power fastelectron beam is supposed to reach the dense target core and ignite the fuel [1]. The typical total energy of such fast electrons may be a large fraction of the total energy of the laser pulse, while the energy per electron is on the order of their oscillation energy in vacuum. Taking as a reference case a beam of fast electrons with an intensity of $10^{18} \mathrm{~W} \mathrm{~cm}^{-2}$ and an energy per electron of $1 \mathrm{MeV}$, one obtains a current density of $10^{12} \mathrm{~A} \mathrm{~cm}^{-2}$. This corresponds to a total current of several mega-amperes through a spot with a radius of $10 \mu \mathrm{m}$, nearly 2 orders of magnitude larger than the Alfvén limit for a monochromatic electron beam. Therefore, the transport of the fast electrons as a collimated beam is only possible by means of a "return" current able to maintain global charge neutrality as well as to compensate locally for the fast-electron current. However, the presence of counter propagating beams carrying equal, but opposite, currents is the typical condition for the development of fast longitudinal electrostatic [two stream (TS)] and/or transverse electromagnetic [current filamentation $(\mathrm{CF})$ ] instabilities [2]. In particular, the CF instability is driven by the magnetic repulsion of opposite currents [3] and is the leading instability in relativistic conditions. It generates strong "quasistatic" magnetic fields. In the laser plasma context, this instability is usually identified with the "Weibel" instability [4], a similar instability driven by temperature anisotropies. In the following we will thus adopt the name Weibel instead of CF instability. In the fast ignition (FI) scenario, it is believed that the Weibel instability should generate ultra strong magnetic fields up to $100 \mathrm{MG}$. The generated magnetic field should therefore play a key role in the transport process and strongly influence the plasma dynamics [5,6]. Moreover, in recent years the CF (Weibel)-TS instability has been actively discussed in the astrophysical context concerning the origin of cos-
PACS numbers: 52.38.Fz, 52.30.Ex, 52.35.Py, 52.65.Kj

mological magnetic fields and different phenomena such as gamma ray bursts, supernovae, and relativistic jets [7].

The Weibel instability in the framework of laser plasma interaction was thoroughly investigated nearly ten years ago [8]. Its analytical theory was developed for homogeneous beams in the cold fluid, fully relativistic regime $[9,10]$ for the general case where the (transverse) Weibel instability is coupled to the (longitudinal) TS instability. Recently, this analysis was extended to the case of a hot beam and/or hot plasma [11], essentially confirming the cold fluid analysis of Ref. [10] where the most unstable mode in the asymmetric case (where a fast, less dense beam is compensated by a slow, denser beam representing the return current) was found to occur for $k_{\|}^{-1} \sim d_{e} \quad\left(k \equiv\right.$ wave vector, $d_{e} \equiv$ electron skin depth). As noted in Ref. [10], in the asymmetric case the most unstable mode is a coupled Weibel-TS mode with a wave vector neither perpendicular nor parallel to the beams; i.e., the Weibel and TS modes cannot be considered as separate modes. In Ref. [10] it was also shown that in the symmetric case (where the beams have equal density and opposite velocities) the most unstable mode occurs for $k_{\|}=0$, corresponding to very long (infinite) magnetic filaments.

In the asymmetric case, collisional effects, not addressed in our model, could, in principle, influence the (much slower) return beam dynamics, and thus the instability evolution. The role of collisions on the instability growth rates in the regime of interest for FI has been recently addressed analytically by a linear fluid normal mode analysis in Ref. [12] and by a linear and quasilinear kinetic approach in the limit of purely transverse modes in Ref. [13]. A full numerical investigation including a collision operator has only recently being started (see, e.g., [14]) and is beyond the scope of this Letter. On the contrary, in the symmetric case where the return beam moves at nearly the same velocity as the injected beam, collisions are not expected to play a major role. 
The possibility of generating electron beams by an ultra intense laser pulse impinging on a critical surface plasma layer has been shown in two dimensions (2D) [15-18] as well as in 3D [19-21] using kinetic particle in cell numerical simulations. Current filamented structures have been found with typical transverse width of the order of $d_{e}$ and elongated in the laser pulse direction starting from the critical surface layer (i.e., much longer than $d_{e}$ ), together with the corresponding magnetic fields. In particular, in Refs. [5,6,21] the filaments seem to self-organize into a ringlike structure.

Energetic electron beams resulting from laser plasma interaction with dense plasmas have been recently claimed to be observed experimentally [21] and some evidence of Weibel-like dynamics when the beam density approaches the background density is discussed. In particular, in Refs. [22,23] a large number of small filaments with typical dimension of the order $d_{e}$ have been reported to form at a faster rate when the beam density is comparable to the background plasma density.

Motivated by the role attributed to the Weibel instability in the electron transport process in overdense plasmas, we discuss the time and length scales which characterize the Weibel-driven 3D structures in the typical conditions of laser plasma interaction. Spatially resonant effects [9] due to the finite transverse size of the beams are explicitly considered. Our aim is to understand whether the observed filamentary current and magnetic field structures can be attributed to the development of the Weibel instability. In our model we consider two basic physical configurations. In the asymmetric case the injected beam propagates in a dense plasma and is neutralized by a much denser, slower beam. In the symmetric case the injected beam has a density comparable to the plasma density and is neutralized by a beam with nearly the same density and (opposite) velocity.

We model the two electron beams as two distinct cold populations and study their interaction using the two fluid, relativistic dissipationless equations given in Ref. [10], Eqs. (1)-(4). These equations are integrated in the 3D numerical domain $-L_{a} / 2<a<L_{a} / 2, a=x, y, z$ and normalized using the plasma frequency and the velocity of light (so that $d_{e}=1$ ). Two inhomogeneous (i.e., of finite transverse size) electron beams are directed in opposite directions along the $z$ axis with constant densities (such that $\left.n_{0,1}+n_{0,2}=1\right)$ and velocities given by $\mathbf{v}_{0,1}=$ $v_{0,1} f(x, y) \mathbf{e}_{z}, \quad \mathbf{v}_{0,2}=-\mathbf{v}_{0,1} n_{0,1} / n_{0,2} \quad$ where $f(x, y)=$ $0.5\left\{\tanh \left[\left(r-r_{0}\right) / R\right]-\tanh \left[\left(r+r_{0}\right) / R\right]\right\}$ and $r=\sqrt{x^{2}+y^{2}}$. At $t=0$ the total current is zero, $n_{0,1} v_{0,1}+n_{0,2} v_{0,2}=0$. The beams have a cylindrical shape of width $2 r_{0}$ with velocity gradient length scale $R$. We perturb the beams by adding a white random noise on the potential vector. Since the instability generates a magnetic field in the $(x, y)$ plane perpendicular to the initial beams, i.e., $B_{z} \ll B_{x}, B_{y}$, we limit our initial noise to the $z$ component of the po- tential vector: $A_{z}(x, y)=\sum_{k_{x}, k_{y}} a_{k_{x}, k_{y}}^{+}\left\{\cos \left[k_{x} x+k_{y} y+\right.\right.$ $\left.\left.\phi\left(k_{x}, k_{y}, z\right)\right]+a_{k_{x}, k_{y}}^{-} \cos \left[k_{x} x-k_{y} y+\phi\left(k_{x}, k_{y}, z\right)\right]\right\}$, where $a_{k_{x}, k_{y}}^{ \pm}=\epsilon /\left(k_{x}^{2}+k_{y}^{2}\right)^{1 / 2}, \phi\left(k_{x}, k_{y}, z\right) \equiv$ random phase.

We present two relativistic, inhomogeneous 3D simulations with $R=1, r_{0}=\pi, \epsilon=10^{-6}, L_{x}=L_{y}=4 \pi$, $L_{z}=8 \pi, N_{x}=N_{y}=256, N_{z}=128$. The first one corresponds to asymmetric and the second to symmetric beams. In both cases, the beams have a finite transverse width of the order of a few $d_{e}$. The parameters are: $n_{0,1}=0.1$, $v_{0,1}=0.95$, (asymmetric case); $n_{0,1}=0.5, v_{0,1}=0.95$, (symmetric case). Since the initial beams are inhomogeneous in the radial direction, the Weibel instability develops a spatial resonance around which the generated magnetic field becomes more and more concentrated. As pointed out in Ref. [9] for a slab 1D configuration, this resonance is located in the region of the beams' velocity gradient and develops from any initial perturbation at nearly the same rate but with a time transient that depends on the gradient of the electron velocities [9]. During this transient the local value of the wavelength in the inhomogeneity direction reduce nearly to $d_{e}$. This resonance occurs independently of whether 2D perturbations (not shown here) or 3D perturbations are considered, and, in the case of cylindrical beams, leads to a ringlike shaped magnetic field.

By performing a number of 2D cylindrical simulations (i.e., in the plane $z=0$ ) with initial perturbation of the form $f(r) \cos (m \theta)$, we found that modes with initial radial wavelength $\lambda_{r}$ larger or much larger than $d_{e}$ develop after a rapid transient with the same growth rate as those with $\lambda_{r} \sim d_{e}$, in agreement with the slab geometry results of Ref. [9], and independently of the azimuthal number, $m=$ $0,1,2, \ldots$, of the initial perturbation. In order to understand which kind of structures are generated by $3 \mathrm{D}$ perturbations, we first investigated whether the presence of the resonance affects the value of the parallel component of the wave vector of the most unstable mode. Roughly speaking, $k_{\|}^{-1} \gg d_{e}$ corresponds to long magnetic filaments, while $k_{\|}^{-1} \sim d_{e}$ corresponds to bubblelike structures since $k_{\perp}^{-1} \sim$ $d_{e}$ in both the asymmetric and symmetric cases. By performing a number of 3D simulations (most of them not shown here), we found no significant change on the most unstable value of $k_{\|}$induced by the resonance with respect to what one could conjecture from the homogeneous results of Ref. [10].

In Figs. 1 and 2 we draw the shaded isocontours of $A_{z}$ in the asymmetric and symmetric case, respectively, in the plane $z=0$ perpendicular to the beams direction. As expected, in the symmetric case the growth rate is larger than in the asymmetric case, as observed in Ref. [23]. We see that in both cases the Weibel generated magnetic field is concentrated in a ring shaped region corresponding to the region of the velocity gradient of the initial beams. In Fig. 3 we show the shaded isosurfaces of $A_{z}$. Positive (negative) 


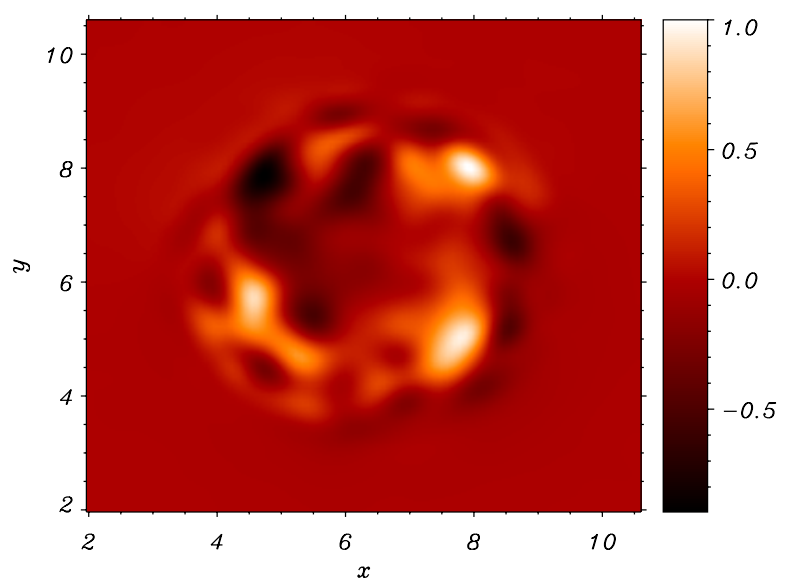

FIG. 1 (color online). Shaded (normalized) isocontours of $A_{z}$ in the $(x, y)$ plane at $z=0, t=30$ in the asymmetric case. Minimum and maximum values are $\sim \pm 0.002$.

values are represented by the darker (lighter) regions, corresponding to a clockwise (counterclockwise) magnetic field. This figure clearly shows that the resulting magnetic field is characterized, in the asymmetric case, by a bubblelike shape with typical length scale of the order of a few $d_{e}$ both in the perpendicular and in the parallel directions, i.e., by $k_{\|}^{-1} \sim k_{\perp}^{-1} \sim d_{e}$. On the other hand, in the symmetric case, the magnetic field structures are strongly anisotropic being elongated in the beams direction, i.e., $k_{\|}^{-1} \gg k_{\perp}^{-1} \sim$ $d_{e}$. Similar results (not presented here) have been obtained, aside from the localization of the magnetic field around the resonance for two initially homogeneous beams.

We define the perpendicular and parallel averaged spectrum of $A_{z}$ as the Fourier spectrum averaged along the $z$ direction and in the $(x, y)$ plane, respectively (and the same for the total density). As expected, in both cases the perpendicular spectra increase at $k_{\perp}<1$ and reach a maximum at $k_{\perp} \sim 1$, corresponding to $\lambda \sim d_{e}$. At larger wave

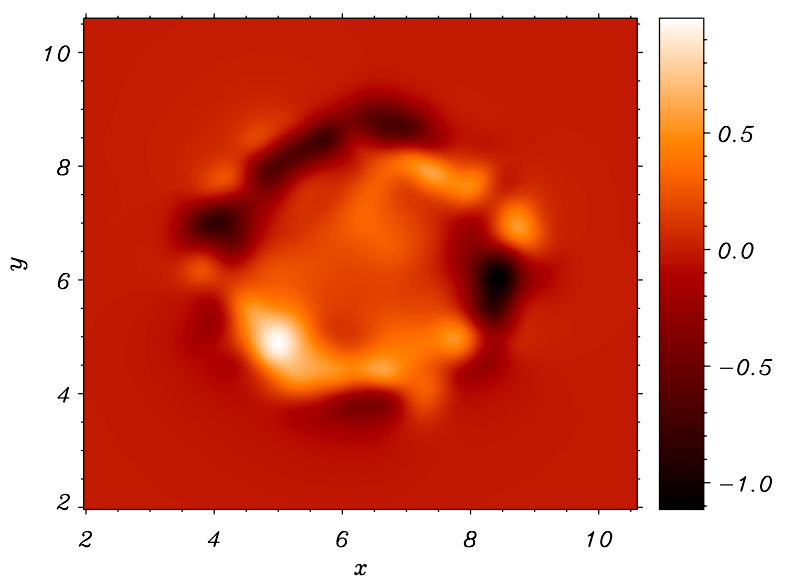

FIG. 2 (color online). Shaded (normalized) isocontours of $A_{z}$ in the $(x, y)$ plane at $z=0, t=25$ in the symmetric case. Minimum and maximum values are $\sim \pm 0.015$. vectors, $k_{\perp} \geq 10$ the spectra decrease due to numerical dissipative effects of a filter, based on a fast-Fouriertransform algorithm, necessary for code stability [24]. Let us now discuss the longitudinal spectra which measure the characteristic length scale of the magnetic "filaments" in the beams' direction. In Fig. 4, first two frames, we see that the $A_{z}$ spectrum peaks are located at $k_{\|} \simeq 0.8$ and at $k_{\|}=0$ for the asymmetric and symmetric case, respectively. This corresponds to a characteristic length scale of the order of a few $d_{e}$ in the asymmetric case, i.e., to a "bubblelike" magnetic structure, and to an "infinite" length scale, i.e., to very long magnetic filaments, in the symmetric case.

By comparing the asymmetric density spectrum, third frame, we observe the same peak at $k_{\|} \simeq 0.8$, but with a spectrum decrease towards $k_{\|}=0$. Moreover, in the symmetric case the density peak is not at $k_{\|}=0$, as observed for $A_{z}$ since at $k_{\|}=0$ the TS contribution is lost and the mode becomes purely electromagnetic. Furthermore, the perpendicular density spectrum (not shown here), driven by nonlinear effects, is more than 2 order of magnitude smaller than the parallel one and rapidly decreases at $k_{\perp}<$ 1 . We therefore conclude that, in this regime, the density fluctuations are mainly driven by the TS contribution. However, density perturbations will strongly change their nature in the further regime when strong nonlinear effects and charge separation effects (induced by the self generated magnetic field) become important.

Our model of a relativistic beam propagating in a dense plasma shows that the Weibel instability generates bubblelike magnetic structures with typical parallel and perpendicular length scales of the order of a few $d_{e}$. This loss of
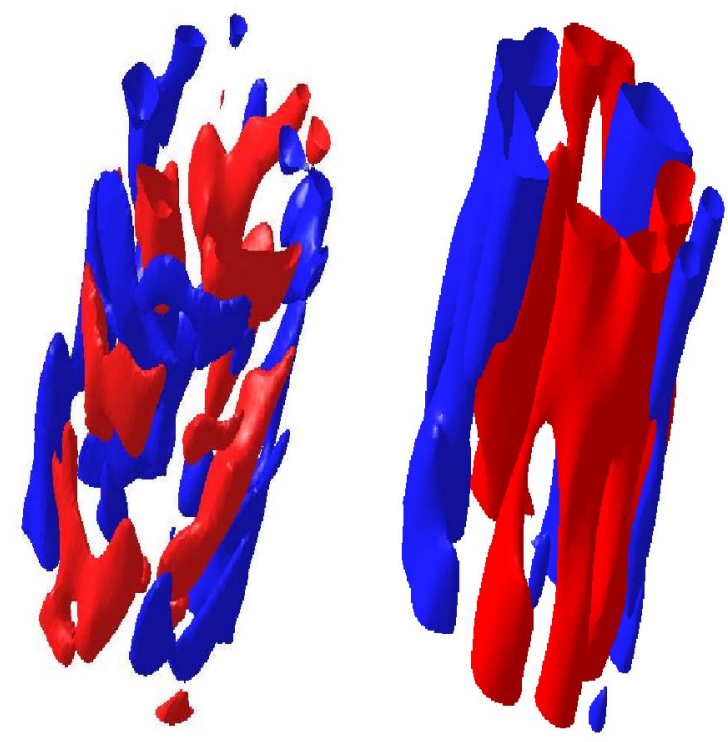

FIG. 3 (color online). Shaded isosurfaces of $A_{z}$ at $t=30$ and $t=25$ in the asymmetric $\left(A_{z} \sim \pm 0.0009\right)$ and symmetric $\left(A_{z} \sim\right.$ $\pm 0.008)$ case, left and right frame, respectively. 

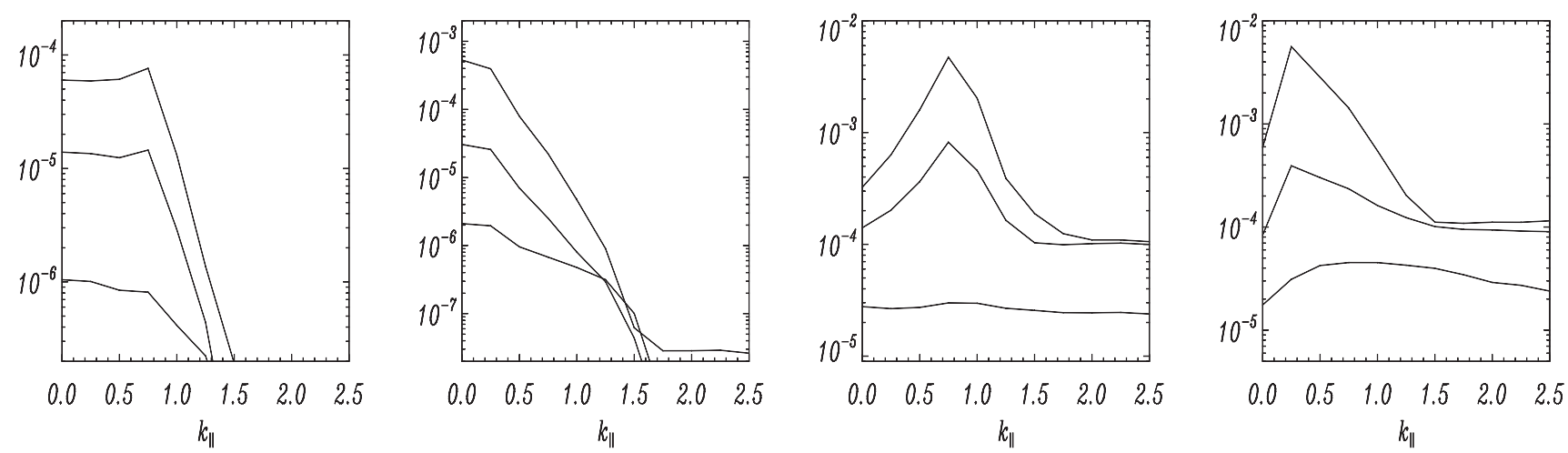

FIG. 4. Parallel averaged spectrum of $A_{z}$ in the asymmetric $(t=15,25,30)$ and in the symmetric $(t=15,20,25)$ case, first and second frame and of the density (same cases), last two frames, respectively. In each frame larger amplitude spectra correspond to increasing times.

spatial coherence is expected to affect the energetic electron transport. However, a quantitative estimate of the impact of such magnetic bubbles on the transport efficiency requires a full kinetic approach (presently under development). On the other hand, when the beam density is comparable to the plasma density, our model of two symmetric relativistic beams shows that the generated magnetic structures have a parallel scale length much longer than $d_{e}$, in agreement with the "Weibel-like" filaments observed in Ref. [22]. The stability of such filaments will be addressed in a forthcoming paper. Finally, we stress that in both the symmetric and asymmetric case, the magnetic field is concentrated in a ringlike structure corresponding to the larger velocity gradients regions due to the development of a spatial resonant-type singularity.

We are pleased to acknowledge A. Macchi for fruitful discussions. This work was supported, in part, by the INFM Parallel Computing Initiative.

[1] M. H. Key, Nature (London) 412, 775 (2001).

[2] Here "longitudinal" and "transverse" indicate the direction parallel and perpendicular to the beams. The same holds for the wave vector components $k_{\|}$and $k_{\perp}$.

[3] G. Kalman, C. Montes, and D. Quemada, Phys. Fluids 11, 1797 (1968); N.W. Albright, Phys. Fluids 13, 1021 (1970).

[4] E. Weibel, Phys. Rev. Lett. 2, 83 (1959).

[5] T. Taguchi, T. Antonsen, C. Liu, and K. Mima, Phys. Rev. Lett. 86, 5055 (2001).

[6] Y. Sentoku, K. Mima, P. Kaw, and K. Nishikawa, Phys. Rev. Lett. 90, 155001 (2003).

[7] C. Hededal and K. Nishikawa, Astrophys. J. Lett. 623, L89 (2005);

R. Schlickeiser, Plasma Phys. Controlled Fusion 47, A205 (2005); J. Wiersma and A. Achterberg, Astron. Astrophys. 428, 365 (2004); K. Nishikawa, P. Hardee, G. Richardson,
R. Preece, H. Sol, and G. Fishman, Astrophys. J. 595, 555 (2003).

[8] G. A. Askar'yan, S. Bulanov, F. Pegoraro, and A. Pukhov, JETP Lett. 60, 251 (1994); T. V. Liseikina, F. Califano, V. Vshivkov, F. Pegoraro, and S. Bulanov, Phys. Rev. E 60, 5991 (1999).

[9] F. Califano, F. Pegoraro, and S. V. Bulanov, Phys. Rev. E 56, 963 (1997).

[10] F. Califano et al., Phys. Rev. E 58, 7837 (1998).

[11] A. Bret, M.-C. Firpo, and C. Deutsch, Phys. Rev. Lett. 94, 115002 (2005).

[12] M. Honda, Phys. Rev. E 69, 016401 (2004).

[13] C. Deutsch, A. Bret, M. Firpo, and P. Fromy, Phys. Rev. E 72, 026402 (2005).

[14] R. Kingham and A. Bella, J. Comput. Phys. 194, 1 (2004).

[15] A. Pukhov and J. Meyer-ter-Vehn, Phys. Rev. Lett. 79, 2686 (1997).

[16] B. Lasinski, A. Langdon, S. Hatchett, M. Key, and M. Tabak, Phys. Plasmas 6, 2041 (1999).

[17] Y. Sentoku, K. Mima, S. Kojima, and H. Ruhl, Phys. Plasmas 7, 689 (2000).

[18] C. Ren et al., Phys. Rev. Lett. 93, 185004 (2004).

[19] Y. Sentoku et al., Phys. Rev. E 65, 046408 (2002).

[20] H. Ruhl, Plasma Sources Sci. Technol. 11, A154 (2002).

[21] R. Jung, J. Osterholz, K. Lowenbruck, S. Kiselev, G. Pretzler, A. Pukhov, O. Willi, S. Kar, M. Borghesi, W. Nazarov, S. Karsch, R. Clarke, and D. Neely, Phys. Rev. Lett. 94, 195001 (2005).

[22] M. Tatarakis, F. Beg, E. Clark, A. Dangor, R. Edwards, R. Evans, T. Goldsack, K. Ledingham, P. Norreys, M. Sinclair, M. Wei, M. Zepf, and K. Krushelnick, Phys. Rev. Lett. 90, 175001 (2003).

[23] M. Wei, F. Beg, E. Clark, A. Dangor, R. Evans, A. Gopal, K. Ledingham, P. McKenna, P. Norreys, M. Tatarakis, M. Zepf, and K. Krushelnick, Phys. Rev. E 70, 056412 (2004).

[24] The filter smooths out progressively the small scales starting from a characteristic length scale $\ell \ll d_{e}=1$, but leaves the large scale dynamics unchanged even on long times: S. K. Lele, J. Comput. Phys. 103, 16 (1992). 